MATHEMATICS OF COMPUTATION

Volume 68, Number 228, Pages 1569-1587

S 0025-5718(99)01110-2

Article electronically published on March 18, 1999

\title{
A CONSTRUCTION OF INTERPOLATING WAVELETS ON INVARIANT SETS
}

\author{
ZHONGYING CHEN, CHARLES A. MICCHELLI, AND YUESHENG XU \\ Dedicated to Shmuel Winograd on the occasion of his sixtieth birthday \\ with friendship and esteem
}

\begin{abstract}
We introduce the concept of a refinable set relative to a family of contractive mappings on a metric space, and demonstrate how such sets are useful to recursively construct interpolants which have a multiscale structure. The notion of a refinable set parallels that of a refinable function, which is the basis of wavelet construction. The interpolation points we recursively generate from a refinable set by a set-theoretic multiresolution are analogous to multiresolution for functions used in wavelet construction. We then use this recursive structure for the points to construct multiscale interpolants. Several concrete examples of refinable sets which can be used for generating interpolatory wavelets are included.
\end{abstract}

\section{INTRODUCTION}

In the recent papers [MX1] and [MX2] we showed how the notion of invariant sets as described in $[\mathrm{H}]$ can be used to construct orthonormal multiwavelet bases on such sets. These wavelets are discontinuous, but nonetheless they have important applications to the numerical solution of integral equations as demonstrated in [CMX] and [MXZ]. See [BCR, D, DPS1, DPS2] for more information about wavelet and multiscale methods for operator equations. For constructions of wavelets and prewavelets, the reader is referred to $[\mathrm{CW}, \mathrm{CDD}$, Da, DJP, DL, M] and references cited therein.

In the present paper we shall explore similar recursive structure for multiscale function representation and approximation by focusing on the analogous situation for interpolation on an invariant set. Thus we seek a mechanism to generate sequences of points which have a multiscale structure that can then be used to efficiently generate interpolating functions. Even in this case it is the theory of

Received by the editor August 11, 1997 and, in revised form, March 3, 1998.

1991 Mathematics Subject Classification. Primary 41A05, 65D15.

Key words and phrases. Refinable sets, set wavelets, interpolating wavelets.

The work on this paper as a whole was partially supported by the National Science Foundation under grant DMS-9504780.

The first author was partially supported by the National Natural Science Foundation of China, the American Lingnan Foundation and the Advanced Research Foundation of Zhongshan University; the work of this author was performed during his visit to North Dakota State University in the academic year 1995-1996.

The third author was also supported in part by the Alexander von Humboldt Foundation. Some of his work was performed when he was visiting RWTH-Aachen, Germany.

(C)1999 American Mathematical Society 
invariant sets $[\mathrm{H}]$ that provides us with an appropriate setting to study this issue and leads us to the notion of a finite subset of a metric space which is refinable relative to a finite collection of contractive mappings on the metric space. A set is called refinable if it is included in its image under a given family of contractive mappings. The sequence of sets which are generated recursively from a refinable set by a set-theoretic multiresolution are analogous to multiresolution of functions used in wavelet construction. Such a set multiresolution will lead us to what we call set wavelets.

This paper is organized as follows. In Section 2 we develop a notion of refinable set, give a complete characterization of refinable sets in a general metric space setting, and illustrate the general characterization with several examples of practical importance. We also show in Section 3 how refinable sets lead to a multiresolution structure relative to set inclusion which is analogous to multiresolution associated with refinable functions. This set-theoretic multiresolution structure leads us to what we call set wavelets, which are generated by a successive application of the contractive mappings to an initial set wavelet. The collection of set wavelets leads us, in particular, in Section 4 to the construction of Lagrange interpolation that has the desired multiscale structure.

\section{REFINABLE SETS}

This section is devoted to a study of refinable sets relative to a family of contractive mappings. A complete characterization of refinable sets will be presented and illustrated by several examples of practical importance.

Following $[\mathrm{H}]$, we let $(X, d)$ be a complete metric space and $\Phi:=\left\{\phi_{\epsilon}: \epsilon \in E_{\mu}\right\}$, $E_{\mu}:=\{0,1, \ldots, \mu-1\}$ be a family of contractive mappings on $X$, where $\mu$ is a positive integer. For any subset $A$ of $X$ and $x \in X$, we define the distance from $x$ to $A$ and the diameter of $A$, respectively, by

$$
\operatorname{dist}(x, A)=\inf \{d(x, y): y \in A\} \text { and } \operatorname{diam}(A)=\sup \{d(x, y): x, y \in A\},
$$

and we introduce the subset of $X$ given by

$$
\Phi(A):=\bigcup_{\epsilon \in E_{\mu}} \phi_{\epsilon}(A) .
$$

The condition of contractivity on the family of mappings $\Phi$ ensures the existence of a $\gamma \in(0,1)$ such that for all subsets $A$ of $X$

$$
\operatorname{diam}\left(\phi_{\epsilon}(A)\right) \leq \gamma \operatorname{diam}(A), \quad \epsilon \in E_{\mu} .
$$

According to $[\mathrm{H}]$, there exists a compact subset $K$ of $X$ such that

$$
\Phi(K)=K,
$$

and $K$ is the unique closed and bounded subset of $X$ which satisfies equation (2.2). The set $K$ is called the invariant set relative to the family of contractive mappings $\Phi$.

Let us recall the construction of the invariant set $K$ given a family of contractive mappings $\Phi$. For every $k \in \mathbb{N}:=\{1,2, \ldots\}$ and $\mathbf{e}_{k}:=\left(\epsilon_{0}, \epsilon_{1}, \ldots, \epsilon_{k-1}\right) \in E_{\mu}^{k}$, where $E_{\mu}^{k}:=E_{\mu} \times \cdots \times E_{\mu}, k$ times, we define the contractive mapping $\phi_{\mathbf{e}_{k}}=$ $\phi_{\epsilon_{0}} \circ \phi_{\epsilon_{1}} \circ \cdots \circ \phi_{\epsilon_{k-1}}$ where $\circ$ denotes the composition of functions on $X$, and we 
let $\Phi_{k}:=\left\{\phi_{\mathbf{e}_{k}}: \mathbf{e}_{k} \in E_{\mu}^{k}\right\}$; in particular, $\Phi_{1}=\Phi$. We let $x_{\mathbf{e}_{k}}$ be the unique fixed point of the mapping $\phi_{\mathbf{e}_{k}}$, that is,

$$
\phi_{\mathbf{e}_{k}}\left(x_{\mathbf{e}_{k}}\right)=x_{\mathbf{e}_{k}},
$$

and set

$$
\mathcal{F}_{k}:=\left\{x_{\mathbf{e}_{k}}: \mathbf{e}_{k} \in E_{\mu}^{k}\right\} .
$$

We define $E_{\mu}^{\infty}$ to be the set of infinite vectors $\mathbf{e}=\left(\epsilon_{0}, \epsilon_{1}, \ldots\right), \epsilon_{i} \in E_{\mu}$, $i \in \mathbb{N}_{0}:=\{0,1, \ldots\}$. With every such vector $\mathbf{e} \in E_{\mu}^{\infty}$ and $k \in N$ we let $\mathbf{e}_{k}=\left(\epsilon_{0}, \epsilon_{1}, \ldots, \epsilon_{k-1}\right) \in E_{\mu}^{k}$. It was shown in $[\mathrm{H}]$ that the limit of $x_{\mathbf{e}_{k}}$ as $k \rightarrow \infty$ exists, and we shall denote this element of the metric space $X$ by $x_{\mathbf{e}}$. In other words, we have that

$$
\lim _{k \rightarrow \infty} x_{\mathbf{e}_{k}}=x_{\mathbf{e}}
$$

The invariant set $K$ is given by either one of the formulas

$$
K=\left\{x_{\mathbf{e}}: \mathbf{e} \in E_{\mu}^{\infty}\right\}
$$

or

$$
K=\overline{\bigcup_{k \in \mathbb{N}} \mathcal{F}_{k}}
$$

The following concept is indispensable to the construction of set wavelets.

Definition 2.1. A subset $V$ of $X$ is said to be refinable relative to the mappings $\Phi$ if $V \subseteq \Phi(V)$.

Observe that the union of any collection of refinable sets is likewise refinable. Moreover, if $V$ is a refinable subset of $X$, then $\Phi_{k}(V)$ is also a refinable subset of $X$ for all $k \in \mathbb{N}$. One of our main objectives is to identify refinable sets of finite cardinality. Before we present a characterization of these sets we look at some examples on the real line which will be helpful to illuminate the general result.

For the metric space $\mathbb{R}$ and an integer $\mu>1$, we consider the mappings

$$
\psi_{\epsilon}(t)=\frac{t+\epsilon}{\mu}, \quad t \in \mathbb{R}, \epsilon \in E_{\mu} .
$$

The invariant set for this family of mappings is the unit interval $[0,1]$. Our first example of a refinable set relative to the family of mappings $\Psi:=\left\{\psi_{\epsilon}: \epsilon \in E_{\mu}\right\}$ given in (2.8) comes next.

Proposition 2.2. The set $U_{0}:=\left\{\frac{j}{k}: j \in E_{k+1}\right\}$ is refinable relative to the mappings $\Psi$.

Proof. For every $j \in E_{k+1}$ we write the integer $\mu j$ uniquely in the form $\mu j=k \epsilon+\ell$, where $\ell-1 \in E_{k}$ and $\epsilon \in \mathbb{N}_{0}$. Since $\mu j \leq \mu k$, we conclude that $\epsilon \in E_{\mu}$. Moreover, we have that $\frac{j}{k}=\psi_{\epsilon}\left(\frac{\ell}{k}\right)$, and so $U_{0}$ is refinable.

In some applications, the exclusion of the endpoints 0 , and 1 from a refinable set is desirable. As an example of this case we present the following fact.

Proposition 2.3. The set $U_{0}=\left\{\frac{j}{k+1}: j-1 \in E_{k}\right\}$ is refinable relative to the mappings $\Psi$ if and only if $\mu$ and $k+1$ are relatively prime. 
Proof. Suppose that $\mu$ and $k+1$ have a common multiple $m>1$, that is, $\mu=m \ell_{1}$ and $k+1=m \ell_{2}$ for some integers $\ell_{1}$ and $\ell_{2}$, and $U_{0}$ is refinable relative to the mappings $\Psi$. Then we have that $\ell_{2}-1 \in E_{k}$ and $\ell_{1} \in E_{\mu}$. Moreover, $\psi_{\ell_{1}}(0)=$ $\frac{\ell_{1}}{\mu}=\frac{\ell_{2}}{k+1}$. This equation implies that $\psi_{\ell_{1}}(0) \in U_{0}$. Since $U_{0}$ is refinable, there exist $\epsilon_{0} \in E_{\mu}$ and $u \in U_{0}$ such that $\psi_{\ell_{1}}(0)=\psi_{\epsilon_{0}}(u)$. It follows from the equation above that $\ell_{1}=u+\epsilon_{0}$. Thus, either $\ell_{1}=\epsilon_{0}$ and $u=0$, or $\epsilon_{0}+1=\ell_{1}$ and $u=1$. In either case we conclude that either 0 or 1 is in $U_{0}$. But this is a contradiction, since $U_{0}$ contains neither 0 nor 1 . Hence, the integers $\mu$ and $k+1$ must be relatively prime.

Conversely, suppose $\mu$ and $k+1$ are relatively prime. For every $j-1 \in E_{k}$ there exist integers $\epsilon$ and $\ell$ such that $j \mu=(k+1) \epsilon+\ell$, where $\ell-1 \in E_{k+1}$. Since $j \mu \leq(k+1) \mu$, it follows that $\epsilon \in E_{\mu}$. Moreover, because $\mu$ and $k+1$ are relatively prime, it must also be the case that $\ell-1 \in E_{k}$. Furthermore, since $\frac{j}{k+1}=\psi_{\epsilon}\left(\frac{\ell}{k+1}\right)$, we conclude that $U_{0}$ is refinable.

Our third special construction of refinable sets $U_{0}$ in $[0,1]$ relative to the mappings $\Psi$ is formed from cyclic $\mu$-adic expansions. To describe this construction we introduce two additional mappings. The first mapping $\pi: E_{\mu}^{\infty} \rightarrow[0,1]$ is defined by

$$
\pi(\mathbf{e}):=\sum_{j \in \mathbb{N}} \frac{\epsilon_{j-1}}{\mu^{j}}, \quad \mathbf{e}=\left(\epsilon_{0}, \epsilon_{1}, \epsilon_{2}, \ldots\right) \in E_{\mu}^{\infty},
$$

and also we write it as $\pi(\mathbf{e})=. \epsilon_{0} \epsilon_{1} \epsilon_{2} \cdots$. This mapping takes an infinite vector $\mathbf{e} \in E_{\mu}^{\infty}$ and associates to it a number in $[0,1]$ whose $\mu$-adic expansion is read off from the components of e. The mapping $\pi$ is not invertible. Referring back to the definition (2.8), we conclude for any $\epsilon \in E_{\mu}$ and $\mathbf{e} \in E_{\mu}^{\infty}$ that $\psi_{\epsilon}(\pi(\mathbf{e}))=$ .$\epsilon \epsilon_{0} \epsilon_{1} \cdots$. We also make use of the "shift" map $\sigma: E_{\mu}^{\infty} \rightarrow E_{\mu}^{\infty}$. Specifically, for an $\mathbf{e}=\left(\epsilon_{0}, \epsilon_{1}, \epsilon_{2}, \ldots\right) \in E_{\mu}^{\infty}$ we set $\sigma(\mathbf{e}):=\left(\epsilon_{1}, \epsilon_{2}, \ldots\right) \in E_{\mu}^{\infty}$. Thus the mapping $\sigma$ discards the first component of $\mathbf{e}$ while the mapping $\psi_{\epsilon}$ restores the corresponding digit, that is,

$$
\psi_{\epsilon_{0}}(\pi \circ \sigma(\mathbf{e}))=\pi(\mathbf{e}) .
$$

For any $k \in \mathbb{N}$ and $\mathbf{e}_{k}=\left(\epsilon_{0}, \epsilon_{1}, \ldots, \epsilon_{k-1}\right) \in E_{\mu}^{k}$ we let. $\bar{\epsilon}_{0} \epsilon_{1} \cdots \epsilon_{k-1}$ denote the number $\pi(\mathbf{e})$ where $\mathbf{e}=\left(\epsilon_{0}, \epsilon_{1}, \ldots\right) \in E_{\mu}^{\infty}$ and $\epsilon_{i+k}=\epsilon_{i}, i \in \mathbb{N}_{0}$. Note that for such an infinite vector e we have that $\sigma^{k}(\mathbf{e})=\mathbf{e}$, where $\sigma^{k}=\sigma \circ \cdots \circ \sigma$ is the $k$-fold composition of $\sigma$, and also the number.$\overline{\epsilon_{0} \epsilon_{1} \cdots \epsilon_{k-1}}$ is the unique fixed point of the mapping $\psi_{\mathbf{e}_{k}}$. Thus, in our previous notation applied to the family of mappings $\Psi$ we have that $x_{\mathbf{e}_{k}}=. \overline{\epsilon_{0} \epsilon_{1} \cdots \epsilon_{k-1}}$.

Proposition 2.4. Choose $k \in \mathbb{N}$ and $\mathbf{e}_{k}=\left(\epsilon_{0}, \epsilon_{1}, \ldots, \epsilon_{k-1}\right) \in E_{\mu}^{k}$ such that at least two components of $\mathbf{e}_{k}$ are different. Let $\mathbf{e}:=\left(\epsilon_{0}, \epsilon_{1}, \ldots\right) \in E_{\mu}^{\infty}$ with $\epsilon_{i+k}=\epsilon_{i}$, $i \in \mathbb{N}_{0}$. Then the set $U_{0}(\pi(\mathbf{e})):=\left\{\pi \circ \sigma^{\ell}(\mathbf{e}): \ell \in E_{k}\right\}$ is refinable relative to the mappings $\Psi$ and has cardinality $\leq k$. Moreover, if $k$ is the smallest positive integer such that $\epsilon_{i+k}=\epsilon_{i}, i \in \mathbb{N}_{0}$ then $U_{0}(\pi(\mathbf{e}))$ has cardinality $k$.

Proof. If $\overline{\epsilon_{0} \epsilon_{1} \cdots \epsilon_{k-1}}=\overline{.} \overline{\epsilon_{0}^{\prime} \epsilon_{1}^{\prime} \cdots \epsilon_{k-1}^{\prime}}$ for $\epsilon_{i}, \epsilon_{i}^{\prime} \in E_{\mu}, i \in E_{k}$, then $\epsilon_{i}=\epsilon_{i}^{\prime}, i \in E_{k}$. Hence, it follows that all the elements of $U_{0}(\pi(\mathbf{e}))$ are distinct. Also, by using (2.9), for any $\ell \in E_{k}$ we have $\pi \circ \sigma^{\ell}(\mathbf{e})=\psi_{\epsilon_{\ell}}\left(\pi \circ \sigma^{\ell+1}(\mathbf{e})\right)$. Note that trivially $\pi \circ \sigma^{\ell+1}(\mathbf{e}) \in U_{0}(\pi(\mathbf{e}))$ for $\ell \in E_{k-1}$ and $\pi \circ \sigma^{k}(\mathbf{e})=\pi(\mathbf{e}) \in U_{0}(\pi(\mathbf{e}))$, and thus $U_{0}(\pi(\mathbf{e}))$ is indeed refinable. 
Various useful examples can be generated from this proposition. We mention the following possibilities for $\mu=2$ :

$$
U_{0}(. \overline{01})=\left\{\frac{1}{3}, \frac{2}{3}\right\}, \quad U_{0}(. \overline{001})=\left\{\frac{1}{7}, \frac{2}{7}, \frac{4}{7}\right\}, \quad U_{0}(. \overline{0011})=\left\{\frac{1}{5}, \frac{2}{5}, \frac{3}{5}, \frac{4}{5}\right\} .
$$

We now present a characterization of refinable sets relative to a given family of contractive mappings $\Phi$ on any metric space $(X, d)$. To state this result, we let $\mathbf{e}_{r, \ell}:=\left(\epsilon_{r}, \epsilon_{r+1}, \ldots, \epsilon_{\ell-1}\right)$ for $r \in E_{\ell+1}$ and use $x_{\mathbf{e}_{r, \ell}}$ to denote the fixed point of the composition mapping $\phi_{\mathbf{e}_{r, \ell}}$, where $\phi_{\mathbf{e}_{r, \ell}}:=\phi_{\epsilon_{r}} \circ \phi_{\epsilon_{r+1}} \circ \cdots \circ \phi_{\epsilon_{\ell-1}}$ when $r \in E_{\ell}$ and $\phi_{\mathbf{e}_{r, \ell}}$ is the identity mapping when $r=\ell$.

Theorem 2.5. Let $\Phi:=\left\{\phi_{\epsilon}: \epsilon \in E_{\mu}\right\}$ be a family of contractive mappings on a complete metric space $X$ and let $V_{0} \subseteq X$ be a nonempty set of cardinality $k \in \mathbb{N}$. Then $V_{0}$ is refinable relative to $\Phi$ if and only if $V_{0}$ has the property that for every $v \in V_{0}$ there exist integers $\ell, m \in E_{k+1}$ with $\ell<m$ and $\epsilon_{i} \in E_{\mu}, i \in E_{m}$, such that $v=\phi_{\mathbf{e}_{0, \ell}}\left(x_{\mathbf{e}_{\ell, m}}\right)$ and the points

$$
v_{r}:=\phi_{\mathbf{e}_{r, \ell}}\left(x_{\mathbf{e}_{\ell, m}}\right) \in V_{0}, \quad r \in E_{\ell}, \quad v_{\ell+r}:=\phi_{\mathbf{e}_{\ell+r, m}}\left(x_{\mathbf{e}_{\ell, m}}\right) \in V_{0}, \quad r \in E_{m-\ell} .
$$

Moreover, in this case, we have that $V_{i}:=\Phi_{i}\left(V_{0}\right) \subseteq K, i \in \mathbb{N}$, and also

$$
V_{0} \subseteq \Phi_{\ell}\left(\mathcal{F}_{m-\ell}\right) \text {. }
$$

Proof. Assume that $V_{0}$ is refinable and $v \in V_{0}$. Let $v_{0}=v$. By the refinability of $V_{0}$, there exist points $v_{j+1} \in V_{0}$, and $\epsilon_{j} \in E_{\mu}$, for $j \in E_{k}$, such that $v_{j}=\phi_{\epsilon_{j}}\left(v_{j+1}\right)$, $j \in E_{k}$. Therefore we have that $v_{r}=\phi_{\mathbf{e}_{r, s}}\left(v_{s}\right), r \in E_{s}, s \in E_{k+1}$. Since the cardinality of $V_{0}$ is $k$, there exist two integers $\ell, m \in E_{k+1}$ with $\ell<m$ for which $v_{\ell}=v_{m}$. Hence, in particular, we conclude that $v_{\ell}=v_{m}=x_{\mathbf{e}_{\ell, m}}$. It follows that

$$
v_{r}=\phi_{\mathbf{e}_{r, \ell}}\left(v_{\ell}\right)=\phi_{\mathbf{e}_{r, \ell}}\left(x_{\mathbf{e}_{\ell, m}}\right), \quad r \in E_{\ell},
$$

and

$$
v_{\ell+r}=\phi_{\mathbf{e}_{\ell+r, m}}\left(v_{m}\right)=\phi_{\mathbf{e}_{\ell+r, m}}\left(x_{\mathbf{e}_{\ell, m}}\right), \quad r \in E_{m-\ell} .
$$

These remarks establish the necessity, and also the fact that $v_{0} \in \Phi_{\ell}\left(\mathcal{F}_{m-\ell}\right) \subseteq K$.

Conversely, let $V_{0}$ be a set of the points with the property and let $v$ be a typical element of $V_{0}$. Then we have that either $v=\phi_{\mathbf{e}_{0, \ell}}\left(x_{\mathbf{e}_{\ell, m}}\right)$ with $\ell>0$, or $v=x_{\mathbf{e}_{0, m}}$ with $\ell=0$. In the first case, since $v=\phi_{\epsilon_{0}}\left(\phi_{\mathbf{e}_{1, \ell}}\left(x_{\mathbf{e}_{\ell, m}}\right)\right)$ and $\phi_{\mathbf{e}_{1, \ell}}\left(x_{\mathbf{e}_{\ell, m}}\right) \in V_{0}$, we have that $v \in \Phi\left(V_{0}\right)$. In the second case, since $x_{\mathbf{e}_{0, m}}$ is the unique fixed point of the mapping $\phi_{\mathbf{e}_{0, m}}$, we write $v=\phi_{\epsilon_{0}}\left(\phi_{\mathbf{e}_{1, m}}\left(x_{\mathbf{e}_{0, m}}\right)\right)$. By our hypothesis, $\phi_{\mathbf{e}_{1, m}}\left(x_{\mathbf{e}_{0, m}}\right) \in$ $V_{0}$, and thus in this case we also have that $v \in \Phi\left(V_{0}\right)$. Therefore, in either case, $v \in \Phi\left(V_{0}\right)$, and so $V_{0}$ is refinable. These comments complete the proof of the theorem.

We next derive two consequences of this observation. To present the first we go back to the definition (2.5) of the point $x_{\mathbf{e}}$, in the metric space $X$, where $\mathbf{e}=$ $\left(\epsilon_{0}, \epsilon_{1}, \ldots\right) \in E_{\mu}^{\infty}$, and observe that when the vector $\mathbf{e}$ is $s$-periodic, that is, its coordinates have the property that $s$ is the smallest positive integer such that $\epsilon_{i}=\epsilon_{i+s}, i \in \mathbb{N}_{0}$, we have that $x_{\mathbf{e}}=x_{\mathbf{e}_{s}}$, where $\mathbf{e}_{s}=\left(\epsilon_{0}, \epsilon_{1}, \ldots, \epsilon_{s-1}\right)$. Conversely, given any $\mathbf{e}_{s} \in E_{\mu}^{s}$, we can extend it as an $s$-periodic vector $\mathbf{e} \in E_{\mu}^{\infty}$ and conclude that $x_{\mathbf{e}}=x_{\mathbf{e}_{s}}$.

Let us observe that the powers of the shift operator $\sigma$ acts on $s$-periodic vectors in $E_{\mu}^{\infty}$ as a cyclic permutation of vectors in $E_{\mu}^{s}$. Also, the $s$-periodic orbits of $\sigma$, that is, vectors $\mathbf{e} \in E_{\mu}^{\infty}$ such that $\sigma^{s}(\mathbf{e})=\mathbf{e}$, are exactly the $s$-periodic vectors 
in $E_{\mu}^{\infty}$. With this viewpoint in mind we can draw the following conclusion from Theorem 2.5.

Theorem 2.6. A finite set $V_{0}$ in a metric space $X$ is refinable relative to the mappings $\Phi$ if and only if for every $v \in V_{0}$ there exists an $\mathbf{e} \in E_{\mu}^{\infty}$ such that $v=x_{\mathbf{e}}$ and $x_{\sigma^{k}(\mathbf{e})} \in V_{0}$ for all $k \in \mathbb{N}$.

Proof. For convenience, we define the notation $\pi^{*}\left(. \epsilon_{0} \epsilon_{1} \epsilon_{2} \ldots\right):=\left(\epsilon_{0}, \epsilon_{1}, \epsilon_{2}, \ldots\right) \in$ $E_{\mu}^{\infty}$ for $\epsilon_{i} \in E_{\mu}$. The proof requires a formula from [H, p.727] which in our notation takes the form

$$
\phi_{\epsilon}\left(x_{\mathbf{e}}\right)=x_{\pi^{*}\left(\psi_{\epsilon}(\pi(\mathbf{e}))\right),}, \quad \epsilon \in E_{\mu}, \quad \mathbf{e} \in E_{\mu}^{\infty},
$$

where $\psi_{\epsilon}$ are the concrete mappings defined by (2.8). Using this formula, the number $\pi(\mathbf{e})$ associated with the vector $\mathbf{e}$ in Theorem 2.5 is identified to be

$$
\pi(\mathbf{e})=. \epsilon_{0} \epsilon_{1} \cdots \epsilon_{\ell-1} \overline{\epsilon_{\ell} \cdots \epsilon_{m-1}} .
$$

An immediate corollary of this result characterizes refinable sets on $\mathbb{R}$ relative to the mappings (2.8).

Theorem 2.7. Let $U_{0}$ be a subset of $\mathbb{R}$ having cardinality $k$. Then $U_{0}$ is refinable relative to the mappings (2.8) if and only if for every point $u \in U_{0}$ there exist integers $\ell, m \in E_{k+1}$ with $\ell<m$, and $\epsilon_{i} \in E_{\mu}, i \in E_{m}$, such that $u=$ .$\epsilon_{0} \cdots \epsilon_{\ell-1} \overline{\epsilon_{\ell} \cdots \epsilon_{m-1}}$ and for any cyclic permutation $\eta_{\ell}, \ldots, \eta_{m-1}$ of $\epsilon_{\ell}, \ldots, \epsilon_{m-1}$ and $r \in E_{\ell}$ the point $\epsilon_{r} \cdots \epsilon_{\ell-1} \overline{\eta_{\ell} \cdots \eta_{m-1}}$ is in $U_{0}$.

It is the vectors $\mathbf{e} \in E_{\mu}^{\infty}$ which are pre-orbits of $\sigma$, that is, for some $k \in \mathbb{N}_{0}$ the vector $\sigma^{k}(\mathbf{e})$ is periodic, which characterize refinable sets. Thus there is an obvious way to build from refinable sets $U_{0}$ relative to the mappings (2.8) on $\mathbb{R}$ refinable sets relative to any finite contractive mappings on a metric space. For example, let $U_{0}$ be a finite subset of cardinality $k$ in the interval $[0,1]$. We require for each number $u$ in this set that there is an $\mathbf{e} \in E_{\mu}^{\infty}$ such that $u=\pi(\mathbf{e})$ and, for every $j \in \mathbb{N}_{0}, \pi\left(\sigma^{j}(\mathbf{e})\right) \in U_{0}$. In other words, $U_{0}$ is refinable relative to the mappings (2.8). We define a set $V_{0}$ in $X$, associated with $U_{0}$, by the formula

$$
V_{0}:=\left\{x_{\mathbf{e}}: \pi(\mathbf{e}) \in U_{0}\right\}
$$

where $x_{\mathbf{e}} \in X$ is defined by the limit (2.5). This set is a refinable subset of $X$ relative to the contractive mappings $\Phi$. We may use this association to construct examples of practical importance in the finite element method and boundary integral equations.

Example. Let $\Delta \subset \mathbb{R}^{2}$ be the triangle with vertices at $y_{0}=(0,0), y_{1}=(1,0)$ and $y_{2}=(0,1)$. Set $y_{3}=(1,1)$ and consider four contractive affine mappings

$$
\phi_{\epsilon}(x):=\frac{1}{2}\left(y_{\epsilon}+(-1)^{\tau(\epsilon)} x\right), \quad \epsilon \in E_{4}, \quad x \in \mathbb{R}^{2},
$$

where $\tau(\epsilon)=0, \epsilon \in E_{3}$ and $\tau(3)=1$. The invariant subset of $\mathbb{R}^{2}$ relative to these mappings is the triangle $\Delta$ and the following sets are refinable:

$$
\begin{aligned}
& \left\{\left(\frac{1}{3}, \frac{1}{3}\right)\right\}, \quad\left\{\left(\frac{1}{7}, \frac{4}{7}\right),\left(\frac{2}{7}, \frac{1}{7}\right),\left(\frac{4}{7}, \frac{2}{7}\right)\right\}, \\
& \left\{\left(\frac{1}{15}, \frac{2}{15}\right),\left(\frac{2}{15}, \frac{4}{15}\right),\left(\frac{4}{15}, \frac{8}{15}\right),\left(\frac{8}{15}, \frac{1}{15}\right)\right\} .
\end{aligned}
$$


Also, we record for any $\mathbf{e}=\left(\epsilon_{0}, \epsilon_{1}, \ldots, \epsilon_{k-1}\right) \in E_{\mu}^{k}$ and $x \in \mathbb{R}^{2}$ that

$$
\phi_{\mathbf{e}_{k}}(x)=\frac{1}{2^{k}}\left[(-1)^{\tau_{k}} x+\sum_{j=0}^{k-1}(-1)^{\tau_{j}} 2^{k-j} y_{\epsilon_{j}}\right],
$$

where $\tau_{j}:=\sum_{\ell=0}^{j-1} \tau\left(\epsilon_{\ell}\right), j \in E_{k+1}$. From this equation it follows that

$$
x_{\mathbf{e}_{k}}=\frac{1}{2^{k}+(-1)^{\tau_{k}+1}} \sum_{j=0}^{k-1}(-1)^{\tau_{j}} 2^{k-j} y_{\epsilon_{j}} .
$$

These formulas can be used to generate the above sets.

\section{Set Wavelets}

In this section, we shall generate a sequence $\mathcal{W}:=\left\{W_{n}: n \in \mathbb{N}_{0}\right\}$ of finite sets of a metric space $X$ which have a wavelet-like multiresolution structure. We call an element of $\mathcal{W}$ a set wavelet, and we shall demonstrate in subsequent sections that set wavelets are crucial for the construction of interpolating wavelets on certain compact subsets of $\mathbb{R}^{d}$.

The generation of set wavelets begins with an initial finite subset $V_{0}:=\left\{v_{j}: j \in\right.$ $\left.E_{m}\right\}$ of distinct points in $X$. We use this subset and the finite set of contractive mappings $\Phi$ to define a sequence of subsets of $X$ given by

$$
V_{i}:=\Phi\left(V_{i-1}\right), \quad i \in \mathbb{N} .
$$

Assume that a compact set $K$ in $X$ is the unique invariant set relative to the mappings $\Phi$. When $V_{0} \subseteq K$, it follows that $V_{i} \subseteq K$ for each $i \in \mathbb{N}$. Furthermore, using the set of contractive mappings $\Phi_{k}:=\left\{\phi_{\mathbf{e}_{k}}: \mathbf{e}_{k} \in E_{\mu}^{k}\right\}$ introduced in the last section, for every subset $A$ of $X$ we define the set

$$
\Phi_{k}(A):=\bigcup_{\mathbf{e}_{k} \in E_{\mu}^{k}} \phi_{\mathbf{e}_{k}}(A),
$$

and so, in particular, $\Phi_{1}(A)=\Phi(A)$. Therefore, the definition (3.1) implies that $V_{i}=\Phi_{i}\left(V_{0}\right), i \in \mathbb{N}$.

The next lemma is useful to us.

Lemma 3.1. Let $\Phi$ be a finite family of contractive mappings on $X$. Assume that $K \subseteq X$ is the invariant set relative to the mappings $\Phi$. If $V_{0}$ is a nonempty finite subset of $X$, then

$$
K \subseteq \overline{\bigcup_{i \in \mathbb{N}_{0}} V_{i}}
$$

where $V_{i}$ is generated by the mappings $\Phi$ by (3.1).

Proof. Let $x \in K$ and $\delta>0$. Since $K$ is a compact set in $X$, we choose an integer $n>0$ such that $\gamma^{n} \operatorname{diam}\left(K \cup V_{0}\right)<\delta$, where $\gamma$ is the contraction parameter appearing in equation (2.1). According to the defining property (2.2) of the set $K$, there exists an $\mathbf{e}_{n} \in E_{\mu}^{n}$ such that $x \in \phi_{\mathbf{e}_{n}}(K) \subseteq \phi_{\mathbf{e}_{n}}\left(K \cup V_{0}\right)$. Since $V_{0}$ is a nonempty set of $X$, there exists a $y \in \phi_{\mathbf{e}_{n}}\left(V_{0}\right) \subseteq \phi_{\mathbf{e}_{n}}\left(K \cup V_{0}\right)$. Moreover, by the contractivity of the family $\Phi$ given by $(2.1)$, we have that

$$
d(x, y) \leq \operatorname{diam} \phi_{\mathbf{e}_{n}}\left(K \cup V_{0}\right) \leq \gamma^{n} \operatorname{diam}\left(K \cup V_{0}\right)<\delta .
$$

This inequality proves the result. 
Proposition 3.2. Let $V_{0}$ be a nonempty refinable set of $X$ relative to a finite family of contractive mappings $\Phi$, and let $\left\{V_{i}: i \in \mathbb{N}_{0}\right\}$ be the collection of sets generated by the definition (3.1). Then

$$
K=\overline{\bigcup_{i \in \mathbb{N}_{0}} V_{i}} .
$$

Proof. This result follows directly from Lemma 3.1 and Theorem 2.5.

This proposition provides another way to construct the unique invariant set $K$ relative to a finite family of contractive mappings $\Phi$. In other words, we start with a refinable set $V_{0}$ and then form $V_{i}, i \in \mathbb{N}$, recursively by (3.1).

We say that a sequence of sets $\left\{A_{i}: i \in \mathbb{N}_{0}\right\}$ is nested (resp., strictly nested) provided that $A_{i-1} \subseteq A_{i}, i \in \mathbb{N}$, (resp., $A_{i-1} \subset A_{i}, i \in \mathbb{N}$ ). The next lemma shows the importance of the notion of the refinable set.

Lemma 3.3. Let $K$ be the invariant set in $X$ relative to a finite family of contractive mappings $\Phi$. Suppose that $K$ is not a finite set and $V_{0}$ is a nonempty finite subset of $X$. Then the collection of sets $\left\{V_{i}: i \in \mathbb{N}_{0}\right\}$ defined by (3.1) is strictly nested if and only if the set $V_{0}$ is refinable relative to $\Phi$.

Proof. Suppose that $V_{0}$ is refinable relative to $\Phi$. Then, it follows by induction on $i \in \mathbb{N}$ that $V_{i-1} \subseteq V_{i}$.

It remains to prove this inclusion is strict for all $i \in \mathbb{N}$. Assume to the contrary that $V_{i-1}=V_{i}$ for some $i \in \mathbb{N}$. By the definition of $V_{i}$, we conclude that $V_{i-1}=V_{j}$ for all $j \geq i$, and thus

$$
\overline{\bigcup_{j \in \mathbb{N}_{0}} V_{j}}=V_{i-1} .
$$

This conclusion contradicts Proposition 3.2 and the fact that $K$ does not have finite cardinality.

When the sequence of sets $\left\{V_{i}: i \in \mathbb{N}_{0}\right\}$ is strictly nested, we let $W_{i-1}:=V_{i} \backslash V_{i-1}$, $i \in \mathbb{N}$, that is, $V_{i}=V_{i-1} \cup^{\perp} W_{i-1}, i \in \mathbb{N}$, where we use the notation $A \cup^{\perp} B$ to denote $A \cup B$ when $A \cap B=\phi$. By Lemma 3.3, if the set $V_{0}$ is refinable relative to $\Phi$, we have that $W_{i} \neq \emptyset, i \in \mathbb{N}$. Similarly, we shall use the notation

$$
\Phi^{\perp}(A):=\bigcup_{\epsilon \in E_{\mu}}{ }^{\perp} \phi_{\epsilon}(A),
$$

when $\phi_{\epsilon}(A) \cap \phi_{\epsilon^{\prime}}(A)=\emptyset, \epsilon, \epsilon^{\prime} \in E_{\mu}, \epsilon \neq \epsilon^{\prime}$. The sets $W_{i}, i \in \mathbb{N}$, give us the decomposition

$$
V_{n}=V_{0} \cup^{\perp}\left(\bigcup_{i \in E_{n}}{ }^{\perp} W_{i}\right) .
$$

The next theorem shows that when the set $W_{0}$ is specified, the sets $W_{i}, i \in \mathbb{N}$, can be recursively constructed and the set $K$ has a decomposition in terms of these sets. This result provides a multiresolution decomposition for the invariant set $K$. For this reason, we call the sets $W_{i}, i \in \mathbb{N}$, set wavelets, the set $W_{0}$ the initial set wavelet and the decomposition of $K$ in terms of $W_{i}, i \in \mathbb{N}$, the set wavelet decomposition of $K$. 
Theorem 3.4. Let $K$ be the invariant set in $X$ relative to a finite family of contractive mappings $\Phi$. Suppose that each of the contractive mappings $\phi_{\epsilon}, \epsilon \in E_{\mu}$, in $\Phi$ has a continuous inverse on $X$ and they have the property that

$$
\phi_{\epsilon}(\text { int } K) \cap \phi_{\epsilon^{\prime}}(\operatorname{int} K)=\emptyset, \quad \epsilon, \epsilon^{\prime} \in E_{\mu}, \epsilon \neq \epsilon^{\prime} .
$$

Let $V_{0}$ be refinable with respect to $\Phi$, and let $W_{0} \subset \operatorname{int} K$. Then

$$
W_{i}=\Phi^{\perp}\left(W_{i-1}\right), \quad i \in \mathbb{N},
$$

and the compact set $K$ has the set wavelet decomposition

$$
K=\overline{V_{0} \cup \perp\left(\bigcup_{n \in \mathbb{N}_{0}}{ }^{\perp} W_{n}\right)} .
$$

Proof. Our hypotheses on the contractive mappings $\phi_{\epsilon}, \epsilon \in E_{\mu}$, guarantee that they are topological mappings. Hence, for any subsets $A$ and $B$ of $X$ and any $\epsilon \in E_{\mu}$ we have

$$
\operatorname{int} \phi_{\epsilon}(A)=\phi_{\epsilon}(\operatorname{int} A)
$$

and

$$
\phi_{\epsilon}(A) \cap \phi_{\epsilon}(B)=\phi_{\epsilon}(A \cap B) .
$$

Let us first establish that when $W_{0} \subset \operatorname{int} K$, the sets $W_{i}, i \in \mathbb{N}$, defined by the recursion (3.4) are all in int $K$. We prove this fact by induction on $i \in \mathbb{N}$. To this end, we suppose that $W_{i-1} \subseteq \operatorname{int} K, i \in \mathbb{N}$. Then, the invariance property (2.2) of $K$ and (3.6) imply that

$$
\Phi\left(W_{i-1}\right) \subseteq \Phi(\operatorname{int} K)=\operatorname{int} \Phi(K)=\operatorname{int} K .
$$

Therefore, we have advanced the induction hypothesis and proved that $W_{i} \subseteq \operatorname{int} K$ for all $i \in \mathbb{N}$. Using the fact that $W_{i} \subseteq \operatorname{int} K$, for any $i \in \mathbb{N}, \epsilon, \epsilon^{\prime} \in E_{\mu}, \epsilon \neq \epsilon^{\prime}$ we conclude from our hypothesis (3.3) that $\phi_{\epsilon}\left(W_{i-1}\right) \cap \phi_{\epsilon^{\prime}}\left(W_{i-1}\right)=\emptyset$, which justifies the " $\perp$ " in formula (3.4). It follows from (3.1), (3.4) and $W_{0}=V_{1} \backslash V_{0}$ that $W_{i} \subseteq V_{i+1}, i \in \mathbb{N}_{0}$.

Next, we wish to confirm that

$$
V_{i+1} \backslash V_{i}=W_{i}, \quad i \in \mathbb{N}_{0} .
$$

Again, we rely upon induction on $i$ and assume that

$$
V_{i} \backslash V_{i-1}=W_{i-1} .
$$

Therefore, we obtain that

$$
V_{i} \cup W_{i}=\Phi\left(V_{i-1}\right) \cup \Phi\left(W_{i-1}\right)=\bigcup_{\epsilon \in E_{\mu}}\left(\phi_{\epsilon}\left(V_{i-1}\right) \cup \phi_{\epsilon}\left(W_{i-1}\right)\right)=\bigcup_{\epsilon \in E_{\mu}} \phi_{\epsilon}\left(V_{i}\right)=V_{i+1},
$$

which implies that $V_{i+1} \backslash V_{i} \subseteq W_{i}$. To confirm that equality holds we observe that

$$
V_{i} \cap W_{i}=\Phi\left(V_{i-1}\right) \cap \Phi\left(W_{i-1}\right)=\bigcup_{\epsilon \in E_{\mu}} \bigcup_{\epsilon^{\prime} \in E_{\mu}}\left(\phi_{\epsilon}\left(V_{i-1}\right)\right) \cap\left(\phi_{\epsilon^{\prime}}\left(W_{i-1}\right)\right) .
$$

For $\epsilon \neq \epsilon^{\prime}$ we can use (3.6) and hypothesis (3.3) to show that $\phi_{\epsilon}(K) \cap \phi_{\epsilon^{\prime}}(\operatorname{int} K)=\emptyset$. To see this, we assume to the contrary that there exists $x \in \phi_{\epsilon}(K) \cap \phi_{\epsilon^{\prime}}(\operatorname{int} K)$. Then there exist $y \in K$ and $y^{\prime} \in \operatorname{int} K$ such that $x=\phi_{\epsilon}(y)=\phi_{\epsilon^{\prime}}\left(y^{\prime}\right)$. Condition (3.3) insures that $y \in K \backslash \operatorname{int} K$. Hence, by equation (3.6), it follows from the 
first equality that $x \in K \backslash \operatorname{int} K$ and from the second equality that $x \in \operatorname{int} K$, a contradiction. Consequently, we have that

$$
\left(\phi_{\epsilon}\left(V_{i-1}\right)\right) \cap\left(\phi_{\epsilon^{\prime}}\left(W_{i-1}\right)\right)=\emptyset .
$$

When $\epsilon=\epsilon^{\prime}$ we use (3.7) to obtain that (3.11) still holds. Hence equation (3.10) implies that $V_{i} \cap W_{i}=\emptyset$. This establishes (3.8), advances the induction hypothesis, and proves the result.

We end this section by considering the following converse question to the one we have considered so far. Given a finite set in a metric space, is it refinable relative to some finite set of contractive mappings? The motivation for this question comes from practical considerations. As is often the case in certain numerical problems associated with interpolation and approximation, we begin on an interval of the real line with prescribed points, for example, Gaussian points or the zeros of Chebyshev polynomials. We then want to find mappings to make these prescribed points refinable relative to them. We shall only address this question in the generality of the space $\mathbb{R}^{d}$ relative to the $\ell_{\infty}$-norm. It is easy to see that, given any subset $V_{0}:=\left\{v_{i}: i \in E_{k}\right\}$ of $\mathbb{R}^{d}$, there is a family of contractive mappings on $\mathbb{R}^{d}$ such that $V_{0}$ is refinable relative to them. For example, the mappings $\phi_{i}(x)=\frac{1}{2}\left(x+v_{i}\right)$, $i \in E_{k}, x \in \mathbb{R}^{d}$, will do, since clearly the fixed point of the mapping $\phi_{i}$ is $v_{i}$ for $i \in E_{k}$. However, almost surely the associated invariant set will have an empty interior, and therefore Theorem 3.4 will not apply. For instance, in the example of a triangle mentioned above, the general prescription applied to the vertices of the triangle will yield the Sierpiński gasket. This invariant set is a Cantor set and is formed by successively applying the maps (2.12) to the triangles; this throws away the middle triangle which is the image of the fourth map used in the example. To overcome this we must add to the above family of mappings another set of contractive mappings "which fill the holes". To describe this process we review some facts about parallelpipeds.

A finite set $\mathcal{I}=\left\{t_{i}: i \in E_{n+1}\right\}$ with $t_{0}<t_{1}<\cdots<t_{n-1}<t_{n}$ is called a partition of the interval $I:=\left[t_{0}, t_{n}\right]$ and divides it into subintervals $I_{i}:=\left[t_{i}, t_{i+1}\right]$, $i \in E_{n}$, where the points in $\mathcal{I} \cap\left(t_{0}, t_{n}\right)$ appear as endpoints of two adjacent subintervals. For every finite set $U_{0}$ of points in $(0,1)$ there exists a partition $\mathcal{I}$ such that the points of $U_{0}$ lie in the interior of the corresponding subintervals. The lengths of these subintervals can be chosen as small as desired.

Likewise, for any two vectors $x:=\left(x_{i}: i \in E_{d}\right), y:=\left(y_{i}: i \in E_{d}\right)$ in $\mathbb{R}^{d}$, where $x_{i}<y_{i}, i \in E_{d}$, which we denote by $x \prec y$ (also $x \preceq y$ when $x_{i} \leq y_{i}, i \in E_{d}$ ), we can partition the set $\bigotimes_{i \in E_{d}}\left[x_{i}, y_{i}\right]$ — called a parallelpiped and denoted by $\langle x, y\rangle$-into (sub) parallelpipeds formed from the partition

$$
\mathcal{I}^{d}:=\bigotimes_{i \in E_{d}} \mathcal{I}_{i}:=\left\{\left(t_{j}: j \in E_{d}\right): t_{i} \in \mathcal{I}_{i}, i \in E_{d}\right\}
$$

where each $\mathcal{I}_{i}$ is a partition of the interval $\left[x_{i}, y_{i}\right], i \in E_{d}$. If $\left\{I_{i, j}: j \in E_{n_{i}}\right\}$ is the set of subintervals associated with the partition $\mathcal{I}_{i}$, then a typical parallelpiped associated with the partition $\mathcal{I}^{d}$ corresponds to a lattice point $i=\left(i_{j}: j \in E_{d}\right)$, where $i_{j} \in E_{n_{j}}, j \in E_{d}$, and it is defined by

$$
\mathcal{I}_{i}=\bigotimes_{j \in E_{d}} I_{i_{j}, j} .
$$


Given any finite set $V_{0} \subset \mathbb{R}^{d}$ contained in the interior of a parallelpiped $P$, we can partition it as above so that the interior of the subparallelpipeds contains the vectors of $V_{0}$. We can, if required, choose the volumes of these subparallelpipeds to be as small as desired.

The set of all parallelpipeds is closed under translation, as the simple rule

$$
\langle x, y\rangle+z:=\{w+z: w \in\langle x, y\rangle\}=\langle x+z, y+z\rangle,
$$

valid for any $x, y, z \in \mathbb{R}^{d}$ with $x \prec y$, demonstrates. To any $x, y \in \mathbb{R}^{d}$ we associate an affine mapping $A$ on $\mathbb{R}^{d}$ defined by the formula

$$
A t:=X t+y, \quad t \in \mathbb{R}^{d},
$$

where $X:=\operatorname{diag}\left(x_{0}, x_{1}, \ldots, x_{d-1}\right)$. Such an affine map takes a parallelpiped bijectively to a parallelpiped (as long as the vector $x$ has no zero components). Conversely, given any two parallelpipeds $P$ and $P^{\prime}$, there exists an affine mapping of the form (3.14) which takes $P$ bijectively to $P^{\prime}$. Moreover, if there exists a $z \in \mathbb{R}^{d}$ such that $P^{\prime}+z \subset \operatorname{int} P$, then $A$ is a contraction relative to the $\ell^{\infty}$-norm on $\mathbb{R}^{d}$ given by

$$
\left\|\left(x_{i}: i \in E_{d}\right)\right\|_{\infty}:=\max \left\{\left|x_{i}\right|: i \in E_{d}\right\} .
$$

For any two parallelpipeds $P=\langle x, y\rangle$ and $P^{\prime}=\left\langle x^{\prime}, y^{\prime}\right\rangle$ with $P^{\prime} \subseteq P$ we can partition their set-theoretic difference into parallelpipeds in the following way. For each $i \in E_{d}$ we partition the interval $\left[x_{i}, y_{i}\right]$ into three subintervals by using the partition $\mathcal{I}_{i}:=\left\{x_{i}, x_{i}^{\prime}, y_{i}^{\prime}, y_{i}\right\}$. The associated partition $\mathcal{I}^{d}$ decomposes $P$ into subparallelpipeds such that one and only one of them corresponds to $P^{\prime}$ itself. In other words, if $\left\{P_{i}: i \in E_{N}\right\}, N=3^{d}$, are the subparallelpipeds which partition $P$ and $P_{N-1}=P^{\prime}$, then we have that

$$
P \backslash P^{\prime}=\bigcup_{i \in E_{N-1}} P_{i} .
$$

We can now state the theorem.

Theorem 3.5. Let $m$ be a positive integer and $V_{0}$ a finite subset of cardinality $m$ in the metric space $\left(\mathbb{R}^{d},\|\cdot\|_{\infty}\right)$. There exists a finite set of contractive mappings $\Phi$ of the form (3.14) such that $V_{0}$ is refinable relative to $\Phi$ and the invariant set for $\Phi$ is a parallelpiped.

Proof. First we put the set $V_{0}$ into the interior of a parallelpiped $P$, which we partition as described above into subparallelpipeds so that the vectors in $V_{0}$ are in the union of the interior of these subparallelpipeds. Specifically, we suppose that

$$
V_{0}=\left\{v_{i}: i \in E_{m}\right\}, \quad P=\bigcup_{i \in E_{M}} P_{i},
$$

with $m<M, v_{i} \in \operatorname{int} P_{i}, i \in E_{m}$, and $V_{0} \cap \operatorname{int} P_{i}=\emptyset, i \in E_{M} \backslash E_{m}$.

For each $i \in E_{m}$ we choose a vector $z_{i}:=\left(z_{i, 0}, z_{i, 1}, \ldots, z_{i, d-1}\right)^{T} \in(0,1)^{d}$ with sufficiently small components $z_{i, j}$ so that the affine mapping

$$
A_{i} t=Z_{i}\left(t-v_{i}\right)+v_{i}, \quad t \in \mathbb{R}^{d},
$$

where $Z_{i}:=\operatorname{diag}\left(z_{i, 0}, z_{i, 1}, \ldots, z_{i, d-1}\right)$, has the property that the parallelpiped $Q_{i}:=$ $A_{i} P$ is contained in $P_{i}$. Since $A_{i} v_{i}=v_{i}, i \in E_{m}$, the set $V_{0}$ is refinable relative to any set of mappings which includes those in (3.15). We wish to append to these $m$ mappings another collection of bijective contractive affine mappings of the type (3.14) so that the extended family has $P$ as an invariant set. 
To this end, for each $i \in E_{m}$ we partition the difference set $P_{i} \backslash Q_{i}$ into parallelpipeds in the manner described above:

$$
P_{i} \backslash Q_{i}=\bigcup_{j \in E_{N-1}} P_{i, j},
$$

where $N=3^{d}$. Thus, we have succeeded in decomposing $P$ into subparallelpipeds so that exactly $m$ of them are the subparallelpipeds $Q_{i}, i \in E_{m}$. In other words, we have

$$
P=\bigcup_{i \in E_{k}} W_{i}
$$

where $m<k$ and $W_{i}=Q_{i}, i \in E_{m}$. Finally, for every $i \in E_{k} \backslash E_{m}$ we choose a bijective contractive affine mapping $A_{i}$ such that $A_{i} P=W_{i}$. This implies that

$$
P=\bigcup_{i \in E_{k}} A_{i} P,
$$

and therefore $P$ is an invariant set relative to the bijective contractive mapping $A_{i}$, $i \in E_{k}$.

In the remainder of this section we look at the above result for the real line and try to economize on the number of affine mappings needed to make a given set $V_{0}$ refinable.

Theorem 3.6. Let $k$ be a positive integer and $V_{0}:=\left\{v_{0}, v_{1}, \ldots, v_{k-1}\right\}$ a subset of distinct points in $[0,1]$ of cardinality $k$. Then, there exists a family of bijective contractive affine mappings $\left\{\phi_{\epsilon}: \epsilon \in E_{\mu}\right\}$ of the type (3.14) for some $2 \leq \mu \leq 4$ when $k=1,2$ and $3 \leq \mu \leq 2 k-1$ when $k \geq 3$ such that $V_{0}$ is refinable relative to these mappings.

Proof. Since the mappings $\phi_{0}(t):=\frac{t}{2}$ and $\phi_{1}(t)=\frac{t+1}{2}$ have the fixed points $t=0$ and $t=1$, respectively, we conclude that when $k=1$ and $V_{0}$ consists of either 0 or 1 and when $k=2$ and $V_{0}$ consists of 0 and 1 , these two mappings are the desired mappings. When $V_{0}$ consists of one interior point $v_{0}$, we need at least three mappings. For example, we choose

$\phi_{0}(t)=\frac{v_{0}}{2} t, \quad \phi_{1}(t)=\frac{1}{2}\left(t-v_{0}\right)+v_{0}$ and $\phi_{2}(t)=\frac{1-v_{0}}{2} t+\frac{v_{0}+1}{2}$, for $t \in[0,1]$.

When $V_{0}$ consists of two interior points of $(0,1)$, we need four mappings constructed by following the spirit of the construction for the case $k \geq 3$ which is given below.

When $k \geq 3$, regardless of the location of the points there exist $2 k-1$ mappings that do the job. We next specifically construct these mappings. Without loss of generality, we assume that $v_{0}<v_{1}<\cdots<v_{k-1}$. We first choose a parameter $\gamma_{1}$ such that

$$
0<\gamma_{1}<\min \left\{\frac{v_{1}-v_{0}}{v_{1}}, \frac{v_{2}-v_{1}}{1-v_{1}}\right\}
$$

and consider the mapping $\phi_{1}(t):=\gamma_{1}\left(t-v_{1}\right)+v_{1}, t \in[0,1]$. Therefore, if we let $\alpha_{1}:=\phi_{1}(0)$ and $\beta_{1}:=\phi_{1}(1)$, then $v_{0}<\alpha_{1}<\beta_{1}<v_{2}$. Next, we let $\gamma_{0}:=$ $\left(\alpha_{1}-v_{0}\right) /\left(1-v_{0}\right)$ and introduce the mapping $\phi_{0}(t):=\gamma_{0}\left(t-v_{0}\right)+v_{0}, t \in[0,1]$. Clearly, by letting $\alpha_{0}:=\phi_{0}(0)$ and $\beta_{0}:=\phi_{0}(1)$, we have $0 \leq \alpha_{0}<\beta_{0}=\alpha_{1}$.

The remaining steps in the construction proceed inductively on $k$. For this purpose, we assume that the affine mapping $\phi_{j-2}$ has been constructed. We let 
$\beta_{j-2}:=\phi_{j-2}(1)$ and define

$$
\phi_{j-1}(t):=\gamma_{j-1}\left(t-v_{j-1}\right)+v_{j-1}, \quad t \in[0,1], \quad j=3,4, \ldots, k-1,
$$

where the parameters $\gamma_{j-1}$ are chosen to satisfy the conditions

$$
0<\gamma_{j-1}<\min \left\{\frac{v_{j-1}-\beta_{j-2}}{v_{j-1}}, \frac{v_{j}-v_{j-1}}{1-v_{j-1}}\right\}, \quad j=3,4, \ldots, k-1 .
$$

It is not difficult to verify that $\phi_{j-1}([0,1]) \subset\left(\beta_{j-2}, v_{j}\right)$, or equivalently $\beta_{j-2}<$ $\alpha_{j-1}<\beta_{j-1}<v_{j}$, by letting $\alpha_{j-1}:=\phi_{j-1}(0)$ and $\beta_{j-1}:=\phi_{j-1}(1)$. Next, we let $\phi_{k-1}(t):=\gamma_{k-1}\left(t-v_{k-1}\right)+v_{k-1}, t \in[0,1]$, where $0<\gamma_{k-1}=\frac{v_{k-1}-\beta_{k-2}}{v_{k-1}}$, and let $\alpha_{k-1}:=\phi_{k-2}(0)$ and $\beta_{k-1}:=\phi_{k-1}(1)$. Then, we have that $\beta_{k-2}=\alpha_{k-1}<$ $\beta_{k-1} \leq 1$. By the construction above, we find two sets of numbers $\left\{\alpha_{i}: i \in E_{k}\right\}$ and $\left\{\beta_{i}: i \in E_{k}\right\}$ that satisfy the condition

$$
0 \leq \alpha_{0}<\beta_{0}=\alpha_{1}<\beta_{1}<\cdots<\beta_{k-2}=\alpha_{k-1}<\beta_{k-1} \leq 1
$$

and the union of the images of $[0,1]$ under mappings $\left\{\phi_{j}: j \in E_{k}\right\}$ is

$$
U:=\bigcup_{j=0}^{k-1}\left[\alpha_{j}, \beta_{j}\right] .
$$

Notice that the set $U$ is not the whole interval $[0,1]$. There are at most $k-1$ gaps which need to be covered. It is straightforward to construct these $k-1$ additional mappings.

The family of mappings of cardinality at most $2 k-1$ that we have constructed above has $[0,1]$ as an invariant set, and $V_{0}$ is a refinable set relative to them.

When the points in a given set have special structure, the number of the mappings may be reduced.

\section{LAGRANGE INTERPOLATING WAVELETS}

We describe in this section a construction of Lagrange interpolating wavelets using the set wavelets constructed previously. For this purpose, we let $X:=\mathbb{R}^{d}$ and assume that $\Phi:=\left\{\phi_{\epsilon}: \epsilon \in E_{\mu}\right\}$ is a family of contractive mappings that satisfies the hypotheses of Theorem 3.4. We also assume that $K \subset \mathbb{R}^{d}$ is the invariant set relative to $\Phi$ with meas $(K \backslash \operatorname{int} K)=0$, where meas $(A)$ denotes the Lebesgue measure of a set $A \subset \mathbb{R}^{d}$. Let $k$ be a positive integer and assume that

$$
V_{0}:=\left\{v_{0}, v_{1}, \ldots, v_{k-1}\right\} \subset \operatorname{int} K
$$

is refinable relative to $\Phi$. Note that in this construction of discontinuous wavelets, we restrict the choice of the points in the set $V_{0}$ to interior points of $K$.

As in [MX1, MX2], we choose a refinable curve $\mathbf{f}:=\left(f_{0}, f_{1}, \ldots, f_{k-1}\right)^{T}: K \rightarrow \mathbb{R}^{k}$ which satisfies a refinement equation

$$
\mathbf{f} \circ \phi_{i}=A_{i} \mathbf{f}, \quad i \in E_{\mu},
$$

for some prescribed $k \times k$ matrices $A_{i}, i \in E_{\mu}$. We remark that if there is $\mathbf{g}: K \rightarrow \mathbb{R}^{k}$ and a $k \times k$ nonsingular matrix $B$ such that $\mathbf{f}=B \mathbf{g}$, then $\mathbf{g}$ is also a refinable curve. We let

$$
\mathbb{F}_{0}:=\operatorname{span}\left\{f_{0}, f_{1}, \ldots, f_{k-1}\right\}
$$

and suppose that $\operatorname{dim} \mathbb{F}_{0}=k$. Furthermore, for any $\mathbf{b}:=\left(b_{0}, b_{1}, \ldots, b_{k-1}\right)^{T} \in \mathbb{R}^{k}$ we require that there exists a unique element $f \in \mathbb{F}_{0}$ such that $f\left(v_{i}\right)=b_{i}, i \in E_{k}$. In 
other words, there exist $k$ elements in $\mathbb{F}_{0}$, which we also denote by $f_{0}, f_{1}, \ldots, f_{k-1}$, such that $f_{i}\left(v_{j}\right)=\delta_{i, j}, i, j \in E_{k}$. When this condition holds we say that $\left\{f_{i}: i \in\right.$ $\left.E_{k}\right\}$ interpolate on the set $V_{0}$ and that $f_{j}$ interpolates at $v_{j}, j \in E_{k}$. Under this condition, any element $f \in \mathbb{F}_{0}$ has a representation of the form

$$
f=\sum_{i \in E_{k}} f\left(v_{i}\right) f_{i} .
$$

A set $V_{0} \subseteq \mathbb{R}^{d}$ is called (Lagrange) admissible relative to $\left(\Phi, \mathbb{F}_{0}\right)$ if it is refinable relative to $\Phi$ and there is a basis of functions $f_{i}, i \in E_{k}$, for $\mathbb{F}_{0}$ which interpolate on the set $V_{0}$. In this section we will always assume that $V_{0}$ is (Lagrange) admissible. We record in the next proposition the simple fact of the Lagrange admissibility of any set of cardinality $k$ for the special case when $\Phi=\Psi$ defined by $(2.8), K=[0,1]$ and $\mathbb{F}_{0}=\mathbb{P}_{k-1}$, the space of polynomials of degree $\leq k-1$.

Proposition 4.1. If $V_{0} \subset[0,1]$ is refinable relative to $\Psi$ and has cardinality $k$, then $V_{0}$ is Lagrange admissible relative to $\left(\Psi, \mathbb{P}_{k-1}\right)$.

Proof. It is a well known fact that the polynomial basis functions satisfy the refinement equation (4.1) with $\phi_{i}=\psi_{i}$ for some matrices $A_{i}$. Hence, this result follows immediately from the unique solvability of the univariate Lagrange interpolation.

In a manner similar to the construction of orthogonal wavelets in [MX1, MX2], we define linear operators $\mathcal{T}_{\epsilon}: L^{\infty}(K) \rightarrow L^{\infty}(K), \epsilon \in E_{\mu}$, by

$$
\left(\mathcal{T}_{\epsilon} x\right)(t):= \begin{cases}x\left(\phi_{\epsilon}^{-1}(t)\right), & t \in \phi_{\epsilon}(K) \\ 0, & t \notin \phi_{\epsilon}(K)\end{cases}
$$

and set

$$
\mathbb{F}_{i+1}=\bigoplus_{\epsilon \in E_{\mu}} \mathcal{T}_{\epsilon} \mathbb{F}_{i}, \quad i \in \mathbb{N}_{0}
$$

This sequence of spaces is nested, i.e., $\mathbb{F}_{i} \subseteq \mathbb{F}_{i+1}, i \in \mathbb{N}_{0}$, and $\operatorname{dim} \mathbb{F}_{i}=k \mu^{i}, i \in \mathbb{N}_{0}$.

We next construct a convenient basis for each of the spaces $\mathbb{F}_{i}$. For this purpose, we let $F_{0}:=\left\{f_{j}: j \in E_{k}\right\}$, where $f_{j}, j \in E_{k}$, interpolate the set $V_{0}$, and

$$
F_{i}:=\bigcup_{\epsilon \in E_{\mu}}{ }^{\perp} \mathcal{T}_{\epsilon} F_{i-1}=\left\{\mathcal{T}_{\epsilon_{0}} \circ \cdots \circ \mathcal{T}_{\epsilon_{i-1}} f_{j}: j \in E_{k}, \quad \epsilon_{\ell} \in E_{\mu}, \quad \ell \in E_{i}\right\}, \quad i \in \mathbb{N} .
$$

Since the functions $f_{j}, j \in E_{k}$, interpolate on the set $V_{0}$, we conclude that the elements in $F_{i}$ interpolate on the set $V_{i}$. In other words, the functions in the set $F_{i}$ satisfy the condition

$$
\left(\mathcal{T}_{\epsilon_{0}} \circ \cdots \circ \mathcal{T}_{\epsilon_{i-1}} f_{j}\right)\left(\phi_{\epsilon_{0}^{\prime}} \circ \cdots \circ \phi_{\epsilon_{i-1}^{\prime}}\left(v_{j^{\prime}}\right)\right)=\delta_{\left(\epsilon_{0}, \ldots, \epsilon_{i-1}, j\right),\left(\epsilon_{0}^{\prime}, \ldots, \epsilon_{i-1}^{\prime}, j^{\prime}\right)},
$$

where we use the notation

$$
\delta_{\mathbf{a}, \mathbf{a}^{\prime}}=\left\{\begin{array}{ll}
1, & \mathbf{a}=\mathbf{a}^{\prime}, \\
0, & \mathbf{a} \neq \mathbf{a}^{\prime},
\end{array} \quad \mathbf{a}, \mathbf{a}^{\prime} \in \mathbb{N}_{0}^{i}, \quad i \in \mathbb{N} .\right.
$$

For ease of notation, we let $\mathbf{e}_{i}:=\left(\epsilon_{0}, \epsilon_{1}, \ldots, \epsilon_{i-1}\right)$ and $\mathcal{T}_{\mathbf{e}_{i}} f_{j}:=\mathcal{T}_{\epsilon_{0}} \circ \cdots \circ \mathcal{T}_{\epsilon_{i-1}} f_{j}$. By (4.3), this function interpolates at $\phi_{\mathbf{e}_{i}}\left(v_{j}\right)$. Moreover,

$$
\mathbb{F}_{i}=\operatorname{span} F_{i}, \quad i \in \mathbb{N}_{0} .
$$


Now, for each $n \in \mathbb{N}_{0}$, we decompose the space $\mathbb{F}_{n+1}$ as the direct sum of the space $\mathbb{F}_{n}$ and its complement space $\mathbb{G}_{n}$, which consists of the elements in $\mathbb{F}_{n+1}$ vanishing at all points in $V_{n}$, that is,

$$
\mathbb{F}_{n+1}=\mathbb{F}_{n} \oplus \mathbb{G}_{n}, \quad n \in \mathbb{N}_{0} .
$$

This decomposition is analogous to the orthorgonal (reps. biorthorgonal) decomposition in the construction of orthorgonal (resp. biorthorgonal) wavelets in [MX1] (resp. [MX2]), and can be viewed as an interpolatory decomposition in the sense to be described below.

We first label the points in the set $V_{n}$ according to the set wavelet decomposition for $V_{n}$ given in Section 2. We assume that the initial set wavelet is given by $W_{0}=\left\{w_{j}: j \in E_{k(\mu-1)}\right\}$, and we let

$$
\begin{gathered}
t_{0, j}:=v_{j}, \quad j \in E_{k} ; \quad t_{1, j}:=w_{j}, \quad j \in E_{k(\mu-1)} ; \\
t_{i, j}:=\phi_{\epsilon_{0}} \circ \phi_{\epsilon_{1}} \circ \cdots \circ \phi_{\epsilon_{i-2}} w_{\ell}, \quad j=\left(\mu^{i-2} \epsilon_{0}+\cdots+\mu \epsilon_{i-3}+\epsilon_{i-2}\right) k(\mu-1)+\ell, \\
\epsilon_{m} \in E_{\mu}, \quad m \in E_{i-1}, \quad \ell \in E_{k(\mu-1)}, \quad i=2,3, \ldots, n .
\end{gathered}
$$

Then, we conclude that $V_{n}=\left\{t_{i, j}: j \in E_{J(i)}, i \in E_{n+1}\right\}$, where

$$
J(i):= \begin{cases}k, & i=0 \\ k(\mu-1) \mu^{i-1}, & i \geq 1\end{cases}
$$

The Lagrange interpolation problem for $\mathbb{F}_{n}$ relative to $V_{n}$ is to find for a vector $\mathbf{b}:=\left(b_{i, j}: j \in E_{J(i)}, i \in E_{n+1}\right)$ an element $f \in \mathbb{F}_{n}$ such that

$$
f\left(t_{i, j}\right)=b_{i, j}, \quad j \in E_{J(i)}, i \in E_{n+1} .
$$

The following fact is useful in this regard.

Lemma 4.2. If $V_{0}$ is (Lagrange) admissible relative to $\left(\Phi, \mathbb{F}_{0}\right)$, then for each $n \in$ $\mathbb{N}_{0}$ the set $V_{n}$ is also (Lagrange) admissible relative to $\left(\Phi, \mathbb{F}_{n}\right)$.

Proof. This result follows immediately from (4.3).

Lemma 4.2 insures that each $f \in \mathbb{F}_{n+1}$ has the representation $f=\mathcal{P}_{n} f+g_{n}$, where $\mathcal{P}_{n} f$ is the Lagrange interpolant to $f$ from $\mathbb{F}_{n}$ relative to $V_{n}$ and $g_{n}=f-\mathcal{P}_{n} f$ is the error of the interpolation. Therefore, we have that

$$
\mathbb{G}_{n}=\left\{g_{n}: g_{n}=f-\mathcal{P}_{n} f, \quad f \in \mathbb{F}_{n+1}\right\} .
$$

The fact that the subspace decomposition (4.5) is a direct sum also follows from equation (4.7) and the unique solvability of the Lagrange interpolation problem (4.6). For this reason, the spaces $\mathbb{G}_{n}$ are called the interpolating wavelet spaces, and in particular, the space $\mathbb{G}_{0}$ is called the initial interpolating wavelet space. A direct computation yields the dimension of $\mathbb{G}_{n}$, namely $\operatorname{dim} \mathbb{G}_{n}=k \mu^{n}(\mu-1)$. Also, we have an interpolating wavelet decomposition for $\mathbb{F}_{n+1}$ :

$$
\mathbb{F}_{n+1}=\mathbb{F}_{0} \oplus \mathbb{G}_{0} \oplus \cdots \oplus \mathbb{G}_{n} .
$$

In the next theorem, we describe a recursive construction for the wavelet spaces $\mathbb{G}_{n}$. To establish the theorem, we need the following lemma regarding the distributivity of the linear operators $\mathcal{T}_{\epsilon}, \epsilon \in E_{\mu}$, relative to a direct sum of two subspaces of $L^{\infty}(K)$.

Lemma 4.3. Let $\mathbb{B}, \mathbb{C} \subset L^{\infty}(K)$ be two subspaces and suppose that $\mathbb{B} \oplus \mathbb{C}$ is their direct sum. Then for each $\epsilon \in E_{\mu}$, we have that $\mathcal{T}_{\epsilon}(\mathbb{B} \oplus \mathbb{C})=\left(\mathcal{T}_{\epsilon} \mathbb{B}\right) \oplus\left(\mathcal{T}_{\epsilon} \mathbb{C}\right)$. 
Proof. It is clear that $\mathcal{T}_{\epsilon}(\mathbb{B} \oplus \mathbb{C})=\left(\mathcal{T}_{\epsilon} \mathbb{B}\right)+\left(\mathcal{T}_{\epsilon} \mathbb{C}\right)$. Therefore, it remains to verify that the sum in the right hand side is a direct sum. To this end, we let $x \in\left(\mathcal{T}_{\epsilon} \mathbb{B}\right) \cap\left(\mathcal{T}_{\epsilon} \mathbb{C}\right)$ and observe that there exist $f \in \mathbb{B}$ and $g \in \mathbb{C}$ such that

$$
x=\mathcal{T}_{\epsilon} f=\mathcal{T}_{\epsilon} g .
$$

By the definition of the operators $\mathcal{T}_{\epsilon}$, we have that $x(t)=0$, for $t \in K \backslash \phi_{\epsilon}(K)$. Now, for each $t \in \phi_{\epsilon}(K)$, there exists $\tau \in K$ such that $t=\phi_{\epsilon}(\tau)$, and thus, using equation (4.9), we observe that

$$
x(t)=f\left(\phi_{\epsilon}^{-1}(t)\right)=f(\tau) \in \mathbb{B}, \quad x(t)=g\left(\phi_{\epsilon}^{-1}(t)\right)=g(\tau) \in \mathbb{C} .
$$

Since $\mathbb{B} \oplus \mathbb{C}$ is a direct sum, we conclude that $x(t)=0$ for $t \in \phi_{\epsilon}(K)$. It follows that $x=0$.

We also need the following fact for the proof of our theorem.

Lemma 4.4. Let $Y \subseteq L^{\infty}(K)$. Then

$$
\mathcal{T}_{\epsilon} Y \cap \mathcal{T}_{\epsilon^{\prime}} Y=\{0\}, \quad \epsilon, \epsilon^{\prime} \in E_{\mu}, \quad \epsilon \neq \epsilon^{\prime} .
$$

Proof. Let $x \in \mathcal{T}_{\epsilon} Y \cap \mathcal{T}_{\epsilon^{\prime}} Y$. There exist $y_{1}, y_{2} \in Y$ such that $x=\mathcal{T}_{\epsilon} y_{1}=\mathcal{T}_{\epsilon^{\prime}} y_{2}$. By the definition of the operators $\mathcal{T}_{\epsilon}$, we conclude from the first equality that $x(t)=0$ for $t \in K \backslash \phi_{\epsilon}(K)$, and from the second that $x(t)=0$ for $t \in K \backslash \phi_{\epsilon^{\prime}}(K)$. Since $\epsilon \neq \epsilon^{\prime}$, we have that meas $\left(\phi_{\epsilon}(K) \cap \phi_{\epsilon^{\prime}}(K)\right)=0$. This implies that $x=0$ a.e. in $K$, and therefore establishes the result in this lemma.

We are now ready to prove the main result of this section.

Theorem 4.5. Let $V_{0}$ be Lagrange admissible relative to $\left(\Phi, \mathbb{F}_{0}\right)$, and let $W_{n}, n \in$ $\mathbb{N}_{0}$, be the set wavelets generated by $V_{0}$. Then it follows that

$$
\begin{gathered}
\mathbb{G}_{0}=\operatorname{span}\left\{\mathcal{T}_{\epsilon} f_{j}: \epsilon \in E_{\mu}, j \in E_{k}, \mathcal{T}_{\epsilon} f_{j} \text { interpolates at } \phi_{\epsilon}\left(v_{j}\right) \in W_{0}\right\}, \\
\mathbb{G}_{n}=\bigoplus_{\epsilon \in E_{\mu}} \mathcal{T}_{\epsilon} \mathbb{G}_{n-1}, \quad n \in \mathbb{N},
\end{gathered}
$$

and $\mathbb{G}_{n}=\operatorname{span} G_{n}$, where

$$
G_{n}:=\left\{\mathcal{T}_{\mathbf{e}_{n+1}} f_{j}: \mathbf{e}_{n+1} \in E_{\mu}^{n+1}, j \in E_{k}, \mathcal{T}_{\mathbf{e}_{n+1}} f_{j} \text { interpolates at } \phi_{\mathbf{e}_{n+1}}\left(v_{j}\right) \in W_{n}\right\}
$$

for $n \in \mathbb{N}_{0}$.

Proof. Let $\mathcal{T}_{\epsilon} f_{j}$ interpolate at $\phi_{\epsilon}\left(v_{j}\right) \in W_{0}$. Then $\mathcal{T}_{\epsilon} f_{j}$ has the property

$$
\left(\mathcal{T}_{\epsilon} f_{j}\right)\left(\phi_{\epsilon^{\prime}}\left(v_{j^{\prime}}\right)\right)=0, \quad \epsilon, \epsilon^{\prime} \in E_{\mu}, \epsilon^{\prime} \neq \epsilon, \quad \text { or } j, j^{\prime} \in E_{k}, j^{\prime} \neq j .
$$

By the definition of the set wavelet, $W_{0}=V_{1} \backslash V_{0}$, we conclude that for all $v_{j^{\prime}} \in V_{0}$ we have $\left(\mathcal{T}_{\epsilon} f_{j}\right)\left(v_{j^{\prime}}\right)=0$. Thus, by the definition of $\mathbb{G}_{0}$, we have that for each point $\phi_{\epsilon}\left(v_{j}\right) \in W_{0}$, the basis function $\mathcal{T}_{\epsilon} f_{j}$ is in $\mathbb{G}_{0}$. Note that the cardinality of $W_{0}$ is given by the formula card $W_{0}=$ card $V_{1}-\operatorname{card} V_{0}=k(\mu-1)$. It follows that the number of the basis functions for which $\mathcal{T}_{\epsilon} f_{j}$ interpolate at $\phi_{\epsilon}\left(v_{j}\right)$ in $W_{0}$ is $k(\mu-1)$, the dimension of $\mathbb{G}_{0}$. Because these $k(\mu-1)$ functions are linearly independent, they constitute a basis for $\mathbb{G}_{0}$.

We next prove equation (4.10) by induction on $n$. For this purpose, we assume that (4.10) holds for $n \leq m$ and consider the case when $n=m+1$. By the definitions of $\mathbb{F}_{m+1}$ and $\mathbb{G}_{m-1}$, we have

$$
\mathbb{F}_{m+1}=\bigoplus_{\epsilon \in E_{\mu}} \mathcal{T}_{\epsilon} \mathbb{F}_{m}=\bigoplus_{\epsilon \in E_{\mu}} \mathcal{T}_{\epsilon}\left(\mathbb{F}_{m-1} \oplus \mathbb{G}_{m-1}\right)
$$


Using Lemma 4.3 and then again the definition of $\mathbb{F}_{m}$, we obtain

$$
\begin{aligned}
\mathbb{F}_{m+1} & =\bigoplus_{\epsilon \in E_{\mu}}\left[\left(\mathcal{T}_{\epsilon} \mathbb{F}_{m-1}\right) \oplus\left(\mathcal{T}_{\epsilon} \mathbb{G}_{m-1}\right)\right] \\
& =\left(\bigoplus_{\epsilon \in E_{\mu}} \mathcal{T}_{\epsilon} \mathbb{F}_{m-1}\right) \oplus\left(\bigoplus_{\epsilon \in E_{\mu}} \mathcal{T}_{\epsilon} \mathbb{G}_{m-1}\right)=\mathbb{F}_{m} \oplus\left(\bigoplus_{\epsilon \in E_{\mu}} \mathcal{T}_{\epsilon} \mathbb{G}_{m-1}\right) .
\end{aligned}
$$

Let

$$
\mathbb{G}:=\bigoplus_{\epsilon \in E_{\mu}} \mathcal{T}_{\epsilon} \mathbb{G}_{m-1}
$$

and assume that $f \in \mathbb{G}$. Then, there exist $g_{0}, \ldots, g_{\mu-1} \in \mathbb{G}_{m-1}$ such that

$$
f=\sum_{\epsilon \in E_{\mu}} \mathcal{T}_{\epsilon} g_{\epsilon}
$$

For each $v \in V_{m}$, there exist $v^{\prime} \in V_{m-1}$ and $\epsilon^{\prime} \in E_{\mu}$ such that $v=\phi_{\epsilon^{\prime}}\left(v^{\prime}\right)$. By the definition of the linear operators $\mathcal{T}_{\epsilon}, \epsilon \in E_{\mu}$, and the fact that $g_{\epsilon} \in \mathbb{G}_{m-1}, \epsilon \in E_{\mu}$, a direct computation leads to the condition that for each $v \in V_{m}$

$$
f(v)=\sum_{\epsilon \in E_{\mu}} \mathcal{T}_{\epsilon} g_{\epsilon}\left(\phi_{\epsilon^{\prime}}\left(v^{\prime}\right)\right)=g_{\epsilon^{\prime}}\left(\phi_{\epsilon^{\prime}}^{-1} \circ \phi_{\epsilon^{\prime}}\left(v^{\prime}\right)\right)=g_{\epsilon^{\prime}}\left(v^{\prime}\right)=0 .
$$

Hence, $\mathbb{G} \subseteq \mathbb{G}_{m}$. On the other hand, it is easy to see that $\operatorname{dim} \mathbb{G}=\operatorname{dim} \mathbb{G}_{m}$, which implies that $\mathbb{G}=\mathbb{G}_{m}$.

To prove the second part of the theorem, it suffices to establish the recursion

$$
G_{n+1}=\bigcup_{\epsilon \in E_{\mu}}{ }^{\perp} \mathcal{T}_{\epsilon} G_{n} .
$$

The " $\perp$ " on the right hand side of this equation is justified by Lemma 4.4. To establish its validity, we let

$$
G:=\bigcup_{\epsilon \in E_{\mu}}{ }^{\perp} \mathcal{T}_{\epsilon} G_{n}
$$

Hence, the set $G$ consists of the elements $\mathcal{T}_{\epsilon_{n+1}} \mathcal{T}_{\mathbf{e}_{n+1}} f_{j}$, where $\mathcal{T}_{\mathbf{e}_{n+1}} f_{j}$ interpolates at $\phi_{\mathbf{e}_{n+1}}\left(v_{j}\right) \in W_{n}, \epsilon_{n+1} \in E_{\mu}$. By Theorem 3.4, we have that

$$
\begin{aligned}
& \left\{\phi_{\mathbf{e}_{n+2}}\left(v_{j}\right):=\phi_{\epsilon_{n+1}} \circ \phi_{\mathbf{e}_{n+1}}\left(v_{j}\right): \epsilon_{n+1} \in E_{\mu}, \phi_{\epsilon_{n+1}}\left(v_{j}\right) \in W_{n}\right\} \\
& \quad \subseteq\left\{\phi_{\mathbf{e}_{n+2}}\left(v_{j}\right) \in W_{n+1}\right\} .
\end{aligned}
$$

Hence, $G_{n+1} \subseteq G$. Since card $G=\operatorname{card} G_{n+1}=\operatorname{card} W_{n+1}$, we conclude that $G=G_{n+1}$.

\section{Theorem 4.6.}

$$
L^{2}(K)=\operatorname{cl}\left(\bigoplus_{n \in \mathbb{N}_{0}} \mathbb{F}_{n}\right)=\operatorname{cl}\left[\mathbb{F}_{0} \oplus\left(\bigoplus_{n \in \mathbb{N}_{0}} \mathbb{G}_{n}\right)\right] .
$$

Proof. Since the mappings $\phi_{\epsilon}, \epsilon \in E_{\mu}$, are contractive, the condition of Theorem 4.7 of [MX2] is satisfied. The finite dimensional spaces $\mathbb{F}_{n}$ appearing here are the same as those generated by the family of mutually orthogonal isometries in [MX2] if we begin with the same initial space $\mathbb{F}_{0}$. Therefore, the first equality holds. An examination of the proof for Theorem 4.7 of [MX2] shows that the same proof proves the second equality. 
As a result of the decomposition obtained in Theorems 4.5 and 4.6, we present a multiscale algorithm for the Lagrange interpolation. To this end, we let $g_{j}$, $j \in E_{k(\mu-1)}$, be a basis for $\mathbb{G}_{0}$, so that

$$
g_{j}\left(t_{0, i}\right)=0, \quad i \in E_{k}, \quad j \in E_{k(\mu-1)}, \quad g_{j}\left(t_{1, j^{\prime}}\right)=\delta_{j, j^{\prime}}, \quad j, j^{\prime} \in E_{k(\mu-1)} .
$$

We label those functions according to points in $V_{n}$ in the following way. Let

$$
\begin{gathered}
g_{0, j}:=f_{j}, j \in E_{k}, \quad g_{1, j}:=g_{j}, \quad j \in E_{k(\mu-1)}, \\
g_{i, j}:=\mathcal{T}_{\epsilon_{0}} \circ \cdots \circ \mathcal{T}_{\epsilon_{i-2}} g_{\ell}, \quad j=\left(\mu^{i-2} \epsilon_{0}+\cdots+\mu \epsilon_{i-3}+\epsilon_{i-2}\right) k(\mu-1)+\ell, \\
\epsilon_{0}, \ldots, \epsilon_{i-2} \in E_{\mu}, \quad \ell \in E_{k(\mu-1)}, \quad i=2,3, \ldots, n .
\end{gathered}
$$

With this labeling, we see that $g_{i, j}\left(t_{i^{\prime}, j^{\prime}}\right)=\delta_{i, i^{\prime}} \delta_{j, j^{\prime}}, j \in E_{J(i)}, j^{\prime} \in E_{J\left(i^{\prime}\right)}, i, i^{\prime} \in$ $E_{n+1}$ with $i^{\prime} \leq i$, and

$$
\mathbb{F}_{n}=\operatorname{span}\left\{g_{i, j}: j \in E_{J(i)}, \quad i \in E_{n+1}\right\} .
$$

Now we express the interpolation projection in terms of this basis. For each $x \in$ $C(K)$, the interpolation projection $\mathcal{P}_{n} x$ of $x$ is given by

$$
\mathcal{P}_{n} x=\sum_{i \in E_{n+1}} \sum_{j \in E_{J(i)}} x_{i, j} g_{i, j} .
$$

The coefficients $x_{i, j}$ in (4.11) can be obtained from the recursive formula

$$
\begin{gathered}
x_{0, j}=x\left(t_{0, j}\right), \quad j \in E_{k}, \\
x_{i, j}=x\left(t_{i, j}\right)-\sum_{i^{\prime} \in E_{i}} \sum_{j^{\prime} \in E_{J\left(i^{\prime}\right)}} x_{i^{\prime}, j^{\prime}} g_{i^{\prime}, j^{\prime}}\left(t_{i, j}\right), \quad j \in E_{J(i)}, i \in E_{n+1} .
\end{gathered}
$$

This recursive formula allows us to efficiently interpolate a given function by functions in $\mathbb{F}_{n}$. When we increase the level from $n$ to $n+1$, we need not recompute the coefficients $x_{i, j}$ for $0 \leq i \leq n$. We describe this important point with the formula $\mathcal{P}_{n+1} x=\mathcal{P}_{n} x+\mathcal{Q}_{n} x$, where $\mathcal{Q}_{n} x \in \mathbb{G}_{n}$ and

$$
\mathcal{Q}_{n} x:=\sum_{j \in E_{J(n+1)}} x_{n+1, j} g_{n+1, j}
$$

The coefficients $x_{n+1, j}$ are computed by the previous recursive formula using the coefficients obtained for the previous levels, that is,

$$
x_{n+1, j}=x\left(t_{n+1, j}\right)-\sum_{i^{\prime} \in E_{n+1}} \sum_{j^{\prime} \in E_{J\left(i^{\prime}\right)}} x_{i^{\prime}, j^{\prime}} g_{i^{\prime}, j^{\prime}}\left(t_{n+1, j}\right) .
$$

Finally, we remark that the idea used in constructing the Lagrange interpolating wavelets can be used to construct the Hermite interpolating wavelets.

\section{REFERENCES}

[BCR] G. Beylkin, R. R. Coifman and V. Rokhlin, Fast wavelet transforms and numerical algorithms I, Comm. Pure and Appl. Math. 44 (1991), 141-183. MR 92c:65061

[CMX] Z. Chen, C. A. Micchelli and Y. Xu, The Petrov-Galerkin methods for second kind integral equations II: Multiwavelet scheme, Advances in Computational Math. 7 (1997), 199-233. CMP 97:13

[CW] C. K. Chui and J. Z. Wang, A cardinal spline approach to wavelets, Proc. Amer. Math. Soc. 113 (1991), 785-793. MR 92b:41019

[CDD] A. Cohen, W. Dahmen and R. DeVore, Multiscale decompositions on bounded domains, Trans. Amer. Math. Soc. (to appear).

[D] W. Dahmen, Wavelet and multiscale methods for operator equations, Acta Numerica 6 (1997), 55-228. MR 98m:65102 
[DPS1] W. Dahmen, S. Proessdorf and R. Schneider, Wavelet approximation methods for pseudodifferential equations I: Stability and convergence, Math. Z. 215 (1994), 583-620. MR 95g:65148

[DPS2] W. Dahmen, S. Proessdorf and R. Schneider, Wavelet approximation methods for pseudodifferential equations II: Matrix compression and fast solution, Advances in Computational Math. 1 (1993), 259-335. MR 95g:65149

[Da] I. Daubechies, Ten Lectures on Wavelets, CBMS-NSF Regional Conference Series in Appl. Math. 61, SIAM, Philadelphia, 1992. MR 93e:42045

[DJP] R. DeVore, B. Jawerth and V. Popov, Compression of wavelet decompositions, Amer. J. Math. 114 (1992), 737-785. MR 94a:42045

[DL] R. DeVore and B. Lucier, Wavelets, Acta Numerica (1992), 1-56. MR 93g:42022

$[\mathrm{H}] \quad$ J. E. Hutchinson, Fractals and self similarity, Indiana Univ. Math. J. 30 (1981), 713-747. MR 82h: 49026

[M] C. A. Micchelli, Using the refinable equation for the construction of prewavelets, Numer. Algorithms 1 (1991), 75-116. MR 93e:65023

[MX1] C. A. Micchelli and Y. Xu, Using the matrix refinement equation for the construction of wavelets on invariant sets, Appl. Comp. Harmonic Anal. 1 (1994), 391- 401. MR 96k: 42044

[MX2] C. A. Micchelli and Y. Xu, Reconstruction and decomposition algorithms for biorthogonal multiwavelets, Multidimensional Systems and Signal Processing 8 (1997), 31-69. MR 98g:94007

[MXZ] C. A. Micchelli, Y. Xu and Y. Zhao, Wavelet Galerkin methods for second-kind integral equations, J. Comp. Appl. Math. 86 (1997), 251-270. CMP 98:06

Department of Scientific Computation, Zhongshan University, Guangzhou 510275, P. R. CHINA

E-mail address: lnsczy@zsulink.zsu.edu.cn

iBm T. J. Watson Research Center, P.O. Box 218, Yorktown Heights, New York 10598-0218

E-mail address: cam@watson.ibm.com

Department of Mathematics, North Dakota State University, Fargo, North Dakota 58105

E-mail address: xu@plains.nodak.edu 